\title{
SISTEMA DE GESTIÓN ISO 9001-2015: TÉCNICAS Y HERRAMIENTAS DE INGENIERÍA DE CALIDAD PARA SU IMPLEMENTACIÓN
}

\section{Management system ISO 9001-2015: techniques and tools of quality engineering for the implementation}

\author{
Fanny Liliana Cruz Medina ${ }^{1}$, Andrea del Pilar López Díaz ${ }^{1}$, Consuelo Ruiz Cardenas ${ }^{2}$ \\ ${ }^{1}$ Universidad Pedagógica y Tecnológica de Colombia, Sogamoso, Maestría en Ingeniería, Colombia. \\ Email: fanny.cruz@uptc.edu.co \\ ${ }^{1}$ Universidad Pedagógica y Tecnológica de Colombia, Sogamoso, Maestría en Ingeniería, Colombia. \\ Email: andrea.lopez03@uptc.edu.co \\ ${ }^{2}$ Universidad Pedagógica y Tecnológica de Colombia, Sogamoso, Escuela de Ingeniería Industrial, Colombia. \\ Email: Consuelo.ruiz@uptc.edu.co
}

(Recibido agosto 26 de 2016 y aceptado diciembre 13 de 2016)

\begin{abstract}
Resumen
El presente artículo muestra una evolución de la calidad en función de la normatividad existente en busca de mejora de procesos, además, como las normas ISO 9000 impactan en el mejoramiento continuo de las organizaciones optimizando los productos ofrecidos al consumidor final. De igual forma el análisis de como la ingeniería de calidad realiza los aportes necesarios en la generación de técnicas y métodos de implementación para seguimiento y control de procesos basados en estadísticas y modelos matemáticos útiles para reducción de costos, reducción de tiempos y mejora de calidad de vida de los empleados, finalmente se realiza un interrelacionar de los requisitos de los que trata la norma ISO 9001:2015 y las herramientas que desde la perspectiva de la ingeniería de la Calidad se pudieran aplicar a fin de garantizar el éxito y sostenibilidad de un Sistema de Gestión Calidad en una organización ya sea de bienes o servicios.
\end{abstract}

Palabras clave: ingeniería de calidad, sistema de gestión de la calidad, control estadístico, mejora de la calidad.

\begin{abstract}
This article shows an evolution of quality management system according to the existing regulations in search process improvement, as well as the ISO 9000 standards impact on continuous improvement of organizations by optimizing the products offered to the final consumer. Similarly analysis and quality engineering makes the necessary inputs in the generation of technical and implementation methods for monitoring and control based on statistics and mathematical models useful for cost reduction, reduction of time and quality improvement processes lives of employees, finally a interrelate with the requirements of ISO 9001:2015 that is standard and tools from the perspective of quality engineering could be applied to ensure the success and sustainability of a system is carried out Quality Management in an organization either goods or services.
\end{abstract}

Key words: Quality Engineering, Management Systems Quality, statistical Control, quality improvement.

\section{INTRODUCCIÓN}

El concepto de la calidad se manifiesta desde la antigüedad, por ejemplo en la construcción de las pirámides de Egipto, en donde la calidad se veía reflejada en la perfección con la que cada piedra fue cortada y encajaba con las otras, hasta formar la compleja estructura piramidal. En la época artesanal, la calidad se concibe como el cumplimiento de los requerimientos establecidos para la personalización de los productos que son manufacturados por expertos maestros. Luego, en la primera mitad del siglo XX con la aparición de las teorías

Cómo citar este artículo:

F.L. Cruz-Medina, A. López-Díaz \& C. Ruíz- Cárdenas, "Sistema De Gestión ISO 9001-2015: Técnicas Y Herramientas De Ingeniería De Calidad Para Su Implementación", Rev. Ingeniería Investigación y Desarrollo, vol. 17 N 1, pp. 59-69, Enero, 2017. 
de Taylor y Ford el concepto de calidad es traducido al concepto de inspección total al final de la línea, donde se verifica que el producto sea capaz de desempeñarse según lo planeado por el diseñador y satisfaga los requisitos de los clientes [1]Cerca al año 1920, Walter Shewart definió la calidad desde dos enfoques, uno subjetivo y otro objetivo; en el segundo, hace referencia al control de la variación de las características de calidad de un producto que son medibles [2], Shewart impulsó la era moderna de la calidad al introducir el control estadístico, dando paso a la primera revolución de la calidad en Estados Unidos durante la segunda guerra mundial; la segunda revolución de la calidad se dio en el año 1950, cuando Williams Edwards Deming, Joseph Juran y Armand Feigenbaum fueron contratados por la Unión de ingenieros y científicos Japoneses (JUSE por sus siglas en inglés), para reconstruir a nivel industrial el caído imperio japonés e impulsar al país como potencia mundial; la tercera revolución se da a finales de 1970 e inicios de 1980, cuando el trabajo de Deming, Juran y Feigenbaum fue reconocido e implementado en Estados Unidos [3]. Deming, señala que la Calidad puede estar definida solamente en términos del agente; es decir de quien la juzgue, en la mente del operario, produce calidad si toma orgullo en su trabajo. La mala calidad, según este agente, significa la pérdida del negocio o de su trabajo. La buena calidad, piensa, mantendrá a la compañía en el negocio. Todo esto es válido en industrias de bienes y servicios. La calidad para el gerente de planta significa obtener las cifras resultantes y conocer las especificaciones. Su trabajo es también el mejoramiento continuo de los procesos y liderazgo [4]; por otro lado Juran estableció que calidad es "adecuación al uso" [5], mientras que Feingebaumg definió un concepto mucho más amplio introduciendo el término calidad total; para quien el objetivo es satisfacer al cliente, y la forma de lograrlo es la mejora continua de la calidad [6].

De otro lado, conforme las organizaciones empezaron a integrar principios de calidad en sus sistemas administrativos, se volvió popular la idea de una gestión o administración de la calidad total o TQM (Total Quality Management, TQM). Más que una disciplina técnica estrecha basada en la ingeniería o la producción, la calidad asumió un nuevo papel que permeó cada aspecto en el funcionamiento de una empresa [1]. A partir de estos diferentes enfoques han surgido varias metodologías como los círculos de calidad total, la reingeniería de procesos, Seissigma, manufactura esbelta, leansigma, entre otras, basadas en técnicas estadísticas y avances en las ciencias conductuales, con el propósito de mejorar la productividad de las empresas [7]. En la década de 1990, especialmente en la industria automotriz se continuo con el énfasis de la calidad, dando lugar a la aparición de la hoy conocida norma ISO 9001 como modelo mundial para los sistema de gestión de la calidad [8]. Esta norma fue creada y adoptada por la Organización Internacional de Normalización (ISO por su sigla en inglés) con el fin de estandarizar los requisitos de calidad para los países europeos dentro del Mercado Común y para quienes querían hacer negocios con esos países, en 1987 [1]. La norma ha sido revisada en 1994, en el año 2000, y nuevamente en el año 2008; en el mes de septiembre del año 2015 la ISO, ha publicado la versión más reciente de esta norma, la cual establece los requisitos para un sistema de gestión de la calidad que puede ser utilizado por cualquier organización, grande $o$ pequeña, independientemente de su campo de acción. Esta norma se basa en una serie de principios de gestión de calidad, incluyendo una fuerte orientación al cliente, la motivación y la implicación de la alta dirección, el enfoque de procesos y la mejora continua. El uso de la norma ISO 9001: 2015 ayuda a asegurar que los clientes obtengan productos consistentes y de buena calidad y servicios, que a su vez trae muchos beneficios para el negocio [9].

Dado lo anterior, el objetivo fundamental de este artículo es desarrollar una revisión de literatura con el fin de determinar cuáles herramientas de la ingeniera de la calidad deberían tenerse en cuenta en los diferentes criterios establecidos en el sistema de gestión de la calidad de la norma ISO 9001:2015, para dar cumplimiento a los propósitos empresariales de mejorar los procesos y lograr la satisfacción de las partes interesadas en la gestión organizacional de la calidad. En la segunda sección se presentaran los resultados de la revisión respecto a los conceptos y la evolución de la ingeniería de la calidad, llegando luego a la identificación de las herramientas de ingeniería de la calidad, en la tercera sección se describirán la conceptualización y estructura del sistema de gestión de la calidad bajo los parámetros de la norma ISO 9001:2015, para lograr en la cuarta parte establecer la relación entre cada numeral de la norma con las diferentes herramientas que pudieran ser aplicadas por cualquier organización. En la parte final se presentan los resultados, conclusiones y recomendaciones para investigaciones posteriores. 


\section{INGENIERA DE LA CALIDAD}

La ingeniería de la calidad es una disciplina que consiste en una serie de técnicas cuantitativas para optimizar productos y procesos de manufactura, creando diseños consistentes o robustos al mínimo costo posible, estos es, diseños afectados mínimamente por fuentes de variabilidad externas o ruidos, las cuales pueden ser de tres tipos: interno por deterioro del producto, externo, causado por el medio ambiente, o por factores humanos, y ruido entre productos o imperfecciones en manufactura [10]; También, en [11], se encuentra una síntesis sobre la aplicación de las técnicas de ingeniería de calidad aplicadas en el diseño de productos, desde lo local hasta un enfoque global, incluyendo estudios de (Clausing,1990), (Lin,990), (Nelson y Winter ,1992),( Hammer y Champy, 1993), (Towner ,1994), (Kackar ,1995), (Box,1996), (Wu,1997), (Ross,1998), (Arthur,1999), (Simón ,1999),( Loasby ,1999), (Hodgson ,1999), entre otros, dando como conceptualización general que la ingeniería de la calidad es el conjunto de actividades para diseñar, mejorar y optimizar procesos, productos y servicios, disminuyendo el tiempo de ciclo, la variabilidad y los costos, con el objetivo de ofrecer lo mejor al mercado.

El control de la calidad, según las Normas Industriales Japonesas, se define como, "Sistema de métodos para la provisión coste-eficaz de bienes o servicios cuya calidad es adecuada a los requisitos del comprador [12]. El control de la calidad según [13] se define como: "el desarrollo, diseño, producción, comercialización y prestación del servicio de productos y servicios con una eficacia del coste y una utilidad óptimas" de tal forma que los clientes comprarán con satisfacción. Para alcanzar estos fines, todas las partes de una empresa (alta dirección, oficina central, fábricas y departamentos individuales tales como producción, diseño, técnico, investigación, planificación, investigación de mercado, administración, contabilidad, materiales, almacenes, ventas, servicio, personal, relaciones laborales y asuntos generales) tienen que trabajar juntos. Todos los departamentos de la empresa tienen que empeñarse en crear sistemas que faciliten la cooperación y en preparar y poner en práctica fielmente las normas internas. Esto sólo puede alcanzarse por medio del uso masivo de diversas técnicas tales como los métodos estadísticos y técnicos, las normas y reglamentos, los métodos computarizados, el control automático, el control de instalaciones, el control de medidas, la investigación operativa, la ingeniería industrial y la investigación de mercado". [14], Define como el proceso de gestión universal para la realización de operaciones con el fin de proporcionar estabilidad para evitar cambio adverso y así lograr mantener el status quo", para mantener la estabilidad, el proceso de control de calidad evalúa el desempeño real, se compara el rendimiento real de los objetivos, y toma una decisión sobre el diferencia.

En 1966, Taguchi introduce el concepto de control estadístico de la Calidad en todo el proceso productivo, realizando así una serie de trabajos en cuanto al control de la producción. En 1984, a raíz del desempeño como consultor de Taguchi, se introduce el término "Método Taguchi", el cual consiste en la aplicación de la función de perdida que mide los costos de la no calidad asumidos por el consumidor. Este concepto llevó a señalar que la Ingeniería de calidad implica la ingeniería del diseño, operaciones de proceso, servicios post ventas, economía y estadística [15], por lo cual sus actividades están enfocadas en la reducción de las pérdidas causadas por la variación, mientras que el control de calidad total consiste esencialmente en desarrollar, controlar y garantizar la calidad de los productos y servicios [16].

Entonces la ingeniería de calidad es atribuida, como técnica al Dr. Genichi Taguchi [17], quien ha desarrollado el concepto desde 1957, cuando realizó una publicación de diseño de experimentos donde propuso la formulación de una matriz ortogonal para la comprobación de experimentos y encontrar así variables clave a lo que el denomino diseño robusto. La ingeniería robusta según [18], es una de las herramientas para la mejora y creación de procesos y productos fiables que se ha mostrado más dinámica desde su definición por el Doctor Taguchi como superación de los anteriores métodos de Diseño de Experimentos. El aspecto al cual se dirige la actividad básica de la ingeniera robusta, es a conseguir productos cuyo diseño los haga menos sensibles a los diversos aspectos que no se puedan controlar y no precisamente extremando los niveles de exigencia en la calidad de las materias primas ni sobredimensionando los cálculos resistentes, sino al contrario, buscando mantener el menor costo y la mayor competitividad.

Otra definición es la Hansen [19], donde denomina control de calidad al conjunto de técnicas y procedimientos de que se sirve la dirección para orientar, supervisar y controlar todas las etapas mencionadas 
hasta la obtención de un producto de la calidad deseada, para él, el control de calidad representa una inversión que, como cualquier otra, debe producir rendimientos adecuados que justifiquen su existencia.

La Ingeniería de calidad es una ciencia interdisciplinaria que se ocupa no sólo de producir productos satisfactorios para los clientes, sino también la reducción de la pérdida total, es decir producir productos de alta calidad a través de un proceso también de calidad [15]. La incertidumbre de los procesos es un aspecto que todas las empresas desean reducir a través de la buena toma de decisiones basadas en procesamiento de datos e información recolectada del sistema empresarial cuya unidad estadística puede ser el proceso productivo o la empresa en su conjunto, con buena información se pueden hacer estudios y análisis de futuro y mejorar los servicios y productos a corto plazo. Según un concepto básico de la gestión de calidad; las decisiones que se tomen sobre el sistema de gestión y sus procesos serán $\tan$ buenos como las mediciones que se realicen [20].

Es aquí donde el uso de técnicas estadísticas a través de los años ha ido tomando fuerza para la mejora continua de los sistemas operacionales permitiendo que a través de los procesos de manufactura se ofrezcan productos fabricados más competitivos [21], al operar bajo principios de cero errores desde el comienzo hasta el final del proceso. La medición de la calidad es efectiva solo cuando se realiza de tal manera que produzca información que la gente pueda entender y utilizar [22], en conexión con esta afirmación, se encuentra un Informe Técnico, ISO/TR 10017, cuyo objeto es ayudar a una organización a identificar las técnicas estadísticas que le puedan ser útiles en el desarrollo, implementación, mantenimiento y mejora de un sistema de gestión de la calidad, en cumplimiento con los requisitos de la Norma ISO 9001 y la Norma ISO 9004.

En dicho informe se relaciona la Estadística descriptiva, Diseño de experimentos, Prueba de hipótesis, Metrología, Análisis de la capacidad de proceso, Análisis de regresión y Análisis de confiabilidad Muestreo, Gráficos de control estadístico de proceso (CEP), Fijación de tolerancias estadísticas y Análisis de series de tiempo [23].

En [24], el autor plantea tres niveles de complejidad, en el primero se enuncian los métodos estadísticos elementales, en el segundo los métodos intermedios y en el tercer nivel métodos avanzados. Los métodos estadísticos elementales, también llamadas siete herramientas son: cuadro de Pareto, diagrama causa efecto, estratificación, hoja de verificación, histograma, diagrama de dispersión, gráficas y cuadros de control. En cuanto a los métodos estadísticos intermedios, se incluyen la teoría del muestreo, la inspección estadísticas por muestreo, diversos métodos de realizar estimaciones y pruebas estadísticas, métodos de utilización de pruebas sensoriales y métodos de diseño de experimentos. De manera similar, los métodos estadísticos avanzados, corresponden al uso de software y computadores para el desarrollo de diseño de experimentos, análisis multivariable y diversos métodos de investigación de operaciones.

En [11], el autor sugiere herramientas de la ingeniería de la calidad aplicados al diseño del producto, tales como Diseño concurrente (DC), Despliegue de la Función de Calidad, análisis del Valor (AV), control estadístico el Proceso (CEP) y análisis de modo de efecto de falla (AMEF).

Todos estos métodos y herramientas son útiles a la hora de establecer, implementar y mantener los sistemas de Gestión de Calidad, ya que tocan todos y cada uno de la factores de la cadena de valor del proceso productivo pasando por la satisfacción de necesidades y expectativas de todos los interesados o mejor conocidos como los stakeholders. Estas herramientas desagregan los objetivos de los sistemas de Gestión basados en las normas de la familia ISO 9000, las cuales corresponden al conjunto de estándares de calidad que prescriben buenas prácticas de calidad [25], este conjunto de normas internacionales que establecen lineamientos, directrices y modelos para la implementación de Sistemas de Gestión de la Calidad, pero que no determinan cómo las empresas deben de obtenerlas [26], es decir, las normas exponen los requisitos que se deben cumplir, en una organización para una certificación en calidad, y las herramientas de la ingeniería de calidad y mejora continua aportan el cómo. La ventaja de las herramientas es que pueden identificar los efectos de los procesos que causan la variabilidad y que resultan en errores y mala calidad; estos pueden determinar de forma fiable la variabilidad anómala en el proceso y contribuir así a mejorar la calidad [27\}. 


\section{SISTEMA DE GESTIÓN DE CALIDAD ISO 9001:2015}

Las normas de sistema de gestión ISO, proporcionan un modelo a seguir para establecer y operar un sistema de gestión, modelo que es construido en consenso internacional, obteniendo como ventaja la experiencia mundial de gestión y las buenas prácticas [28]. La familia de normas ISO 9000, trata diversos aspectos de la gestión de la calidad y proporcionan orientación y herramientas para las empresas y organizaciones que quieren asegurarse de que sus productos y servicios cumplen consistentemente con los requerimientos del cliente, y que la calidad se mejora constantemente. Esta familia de normas incluye la norma ISO 9000, la norma ISO 9001, ISO 9004 y la norma ISO 19011. Actualmente la norma ISO 9000 en versión 2015 presenta los principios, términos y definiciones básicas de la calidad, la norma ISO 9001:2015 establece los requisitos de un sistema de gestión de la calidad, siendo este el único estándar certificable de la familia ISO 9000, de igual manera la norma ISO 9004: 2009, se centra en cómo hacer que un sistema de gestión de calidad más eficiente y eficaz, por su parte la norma ISO 19011: 2011, presenta una guía sobre las auditorías internas y externas de los sistemas de gestión [28].

Un Sistema de Gestión de Calidad (SGC) basado en la norma ISO 9001, proporciona una base sólida para la construcción de un programa de Gestión total de la calidad (TQM por sus siglas en inglés), siendo este estándar, el primer gran paso en la implementación de un programa TQM, facilitando el camino hacia la mejora continua [29]. En la norma ISO 9000:2015, se define el sistema de gestión de la calidad como el conjunto de elementos de una organización que están interrelacionados o que interactúan para establecer políticas, objetivos, y procesos de calidad para alcanzar dichos objetivos de calidad [30]. El sistema de gestión de calidad se encuentra enmarcado en siete principios fundamentales: 1) el enfoque al cliente, 2) el liderazgo, 3 ) el compromiso de las personas, 4) el enfoque en procesos, 5) la mejora, 6) la toma de decisiones basada en la evidencia, y 7) la gestión de las relaciones [30]. En la figura No 1, se muestra la estructura de la Norma ISO 9001:2015 con relación al ciclo PHVA: Planificar - Hacer - Verificar - Actuar.

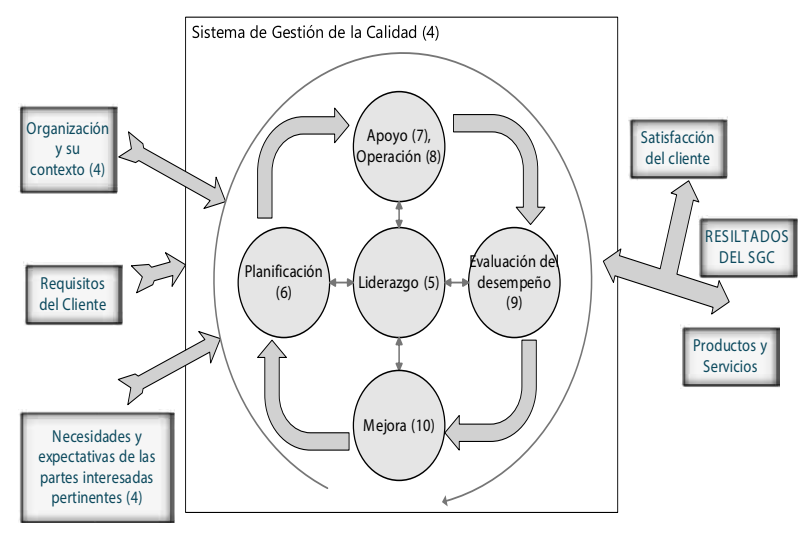

Figura No 1. Estructura de la Norma ISO 9001:2015 con relación al ciclo PHVA. Fuente: [31]

En [31], se incluyen cambios como la definición de la estructura de alto nivel, con el fin de lograr la alineación con otros sistema de gestión y se da una mayor relevancia a la gestión del riesgo teniendo en cuenta los principios y el proceso establecido en la NTC ISO 31000, en cuanto a la identificación del riesgo y la determinación de acciones para abordar los riesgos [32]; se determina una nueva ordenación del sistema de gestión de la calidad ISO 9001, la cual plantea diez numerales a tener en cuenta. Del numeral 1 al 3 se encuentra: 1) el alcance, 2) las referencias normativas y 3 ) la referencia a términos y definiciones de la norma ISO 9000:2015. Los numerales 4 al 10 presentan los requisitos o criterios a establecer, implementar y mantener en el sistema de gestión de la calidad de cualquier organización. A continuación en la tabla 1 , se presentan y explican dichos numerales:

Tabla 1. Requisitos cuatro al ocho de la Norma ISO 9001:2015.

\begin{tabular}{|c|c|}
\hline NUMERAL & DESCRIPCIÓN \\
\hline $\begin{array}{l}\text { 4) Contexto de la } \\
\text { organización: }\end{array}$ & $\begin{array}{l}\text { La organización debe determinar las } \\
\text { cuestiones internas y externas, las partes } \\
\text { interesadas, los requisitos de tales partes } \\
\text { interesadas así como el alcance y el sistema } \\
\text { de gestión de la calidad y sus procesos. }\end{array}$ \\
\hline 5) Liderazgo: & $\begin{array}{l}\text { La organización debe demostrar el liderazgo } \\
\text { y compromiso con el SGC a través de la } \\
\text { rendición de cuentas, comprendiendo } \\
\text { e impulsando el enfoque al cliente, } \\
\text { estableciendo y comunicando la política de } \\
\text { calidad y definiendo roles, responsabilidades } \\
\text { y autoridades en la organización. }\end{array}$ \\
\hline
\end{tabular}

6) Planificación: Describe requisitos a cumplir en cuanto a la definición de las acciones para abordar riesgos y oportunidades, los objetivos de la calidad y la planificación para lograrlos y la planificación de los cambios 


\begin{tabular}{ll}
\hline \multicolumn{1}{c}{ NUMERAL } & \multicolumn{2}{c}{ DESCRIPCIÓN } \\
\hline 7) Apoyo: & Presenta los criterios en los cuales \\
& la organización debe determinar y \\
& proporcionar los recursos necesarios \\
& para el establecimiento, implementación, \\
& mantenimiento y mejora continua del SGC, \\
& tales recursos deben considerar las personas, \\
& la infraestructura, el ambiente para la \\
& operación de los procesos, los recursos de \\
& seguimiento y medición y los conocimientos \\
& de la organización; este numeral Incluye \\
& además la determinación y aseguramiento \\
& de la competencia, la toma de conciencia, \\
& la comunicación y la creación y control de la \\
& información documentada
\end{tabular}

8) Operación: Se concentra en las acciones para la planificación, implementación y control de los procesos para la provisión de productos y servicios, a través de la planificación y control operacional, de la comunicación, determinación y revisión de los requisitos para los productos y servicios, el diseño y desarrollo de los productos y servicios, el control de los procesos, productos y servicios suministrados externamente, la producción y la provisión del servicio, la liberación de los productos y servicios y el control de las salidas no conformes.

9) Evaluación del Incluye los criterios para realizar seguimiento, desempeño: medición, análisis y evaluación al SGC, a sus procesos, productos y servicios, de igual forma señala la determinación del proceso de auditoría interna y de revisión por la dirección.

10) Mejora: Busca que la organización determine y seleccione las oportunidades de mejora e implemente cualquier acción necesaria para cumplir los requisitos del cliente y aumentar la satisfacción del cliente, a través de la determinación de acciones de corrección y la identificación de No conformidades y acciones correctivas, así como la mejora continua de la conveniencia, adecuación y eficacia del sistema de gestión de la calidad.

A pesar que la certificación en la norma ISO 9001:2015, se entiende que debería ser un paso inicial hacia otras estrategias de calidad total, por sí sola la certificación no parece contribuir a las mejoras en los resultados empresariales [33], por otro lado [26], plantean que la norma ISO es un conjunto de estándares de calidad que establecen buenas prácticas de calidad, sin embargo este modelo no determina cómo las empresas deben establecerlas e implementarlas, por lo que se argumenta la necesidad de que exista una fuerte cultura de calidad en la empresa certificada para que esta iniciativa implique mejoras en los resultados empresariales [34]. De allí que se plantea la necesidad de estudiar y analizar con mayor profundidad el área de la ingeniería de la calidad, con el fin de identificar y alinear herramientas apropiadas para la creación de la cultura de la calidad en una organización que implemente el sistema de gestión de la calidad bajo la norma ISO 9001:2015

\section{INGENIERIA DE LA CALIDAD APLICABLE AL ÉXITO DE LOS SISTEMAS DE GESTION LA CALIDAD ISO 9001:2015}

Luego de la revisión de la evolución de la calidad, el alcance de los Sistemas de Gestión Calidad, y de la Ingeniería de la Calidad, se dedicará esta sección a interrelacionar los requisitos de los que trata la norma ISO 9001: 2015 en sus numerales 8. Operación, 9. Evaluación de Desempeño y 10. Mejora Continua, y las herramientas que desde la perspectiva de la ingeniería de la Calidad se pudieran aplicar a fin de garantizar el éxito y sostenibilidad de un Sistema de Gestión Calidad en una organización ya sea de bienes o servicios. Es importante señalar, que para efectos de este artículo se toman únicamente los numerales 8,9 y 10 de la norma ISO 9001:2015, dado que se puede evidenciar una mayor necesidad de uso de datos cuantitativos para su gestión.

En la tabla 2. Se presenta la correspondencia entre numerales de la norma ISO 9001:2015 y herramientas estadísticas, tomando como referencia los criterios establecidos en el informe técnico ISO/TR 10017/2003. Orientación para el uso de técnicas estadísticas, las herramientas definidas por Kauro Ishikawa en su libro ¿Qué es el control total de la calidad?, y otros autores. 
Tabla 2. Correspondencia entre los numerales de la norma ISO 9001:2015 y las técnicas y herramientas de Ingeniería de Calidad.

\begin{tabular}{l} 
Numeral ISO 9001:2015 \\
\hline 8. Operación \\
8.1. Planificación y control operacional: La organización debe planificar \\
implementar y controlar los procesos necesarios para cumplir los requisitos \\
para la provisión de productos y servicios, y para implementar las acciones \\
determinadas en la planificación.
\end{tabular}

8.2. Requisitos para los productos y servicios: Incluye los requisitos con la comunicación con el cliente, la determinación, revisión y cambios de los requisitos para los productos y servicios,

8.3. Diseño y desarrollo de los productos y servicios: La organización debe establecer, implementar y mantener un proceso de diseño y desarrollo que incluya la planificación, las entradas, los controles, las salidas y los cambios del diseño y desarrollo.

8.4. Control de los procesos, productos o servicios suministrados externamente: La organización debe asegurarse de que los procesos, productos y servicios suministrados externamente son conformes a los requisitos, determinando el tipo y alcance del control y la información para los proveedores externos.

8.5. Producción y provisión del servicio: La organización debe implementar la producción y provisión del servicio bajo condiciones controladas, debe utilizar los métodos apropiados para la identificación y trazabilidad de los productos y servicios, debe cuidar la propiedad perteneciente al cliente, debe preservar las salidas durante la producción y prestación del servicio, debe cumplir los requisitos para las actividades posteriores a la entrega asociadas con los productos y servicios, y debe controlar los cambios para la producción y prestación de servicios.

8.6. Liberación de productos y servicios: La organización debe implementar las disposiciones planificadas, en las etapas adecuadas, para verificar que se cumplen los requisitos de los productos y servicios.

8.7. Control de los procesos no conformes a productos y servicios: La organización debe asegurarse de que las salidas que no sean conformes con sus requisitos se identifican y se controlan para prevenir su uso o entrega no intencionada, y mantener información documentada de la no conformidad, las acciones tomadas, las concesiones obtenidas y la autoridad que decide la acción frente a la no conformidad.

\section{Evaluación del desempeño}

9.1. Seguimiento, medición, análisis y evaluación: La organización debe determinar qué necesita seguimiento y medición, los métodos de seguimiento, medición, análisis y evaluación necesarios para asegurar resultados válidos., debe realizar el seguimiento de las percepciones de los clientes del grado en que se cumplen sus necesidades y expectativas. La organización debe analizar y evaluar los datos y la información apropiados que surgen por el seguimiento y la medición.

9.2. Auditoría interna: La organización debe llevar a cabo auditorías internas a intervalos planificados para proporcionar información acerca de si el SGC es conforme a los requisitos de la organización y de la norma y se implementa y mantiene.

9.3. Revisión por la dirección: La alta dirección debe revisar el SGC a intervalos planificados para asegurarse de sus conveniencia, adecuación, eficacia y alineación continuas de las dirección estratégica de la organización.
Despliegue de la función de la calidad (QFD), Análisis de Modo de Efectos y Fallos (AMEF), Muestreo e inspección, hojas de verificación, cartas de control, series de tiempo.

QFD, metrología, análisis de la capacidad del proceso, muestreo, fijación de tolerancia estadística, hoja de verificación.

QFD, AMEF, diseño de experimentos, prueba de hipótesis, metrología, análisis de regresión, análisis de confiabilidad, muestreo, simulación, análisis de series de tiempo, Diseño Concurrente, Análisis de Valor, hoja de verificación.

Muestreo, Inspección, análisis de la calidad del proceso, prueba de hipótesis; metrología; análisis de regresión; análisis de confiabilidad; diseño de experimentos, hoja de verificación, diagrama de Pareto.

Investigación de Operaciones, metrología, análisis de la capacidad del proceso, análisis de regresión; análisis de confiabilidad, muestreo, gráficos de Control Estadístico de Proceso, análisis de series de tiempo, hoja de verificación.

Metrología, análisis de la capacidad del proceso, análisis de regresión, muestreo, gráficos de Control Estadístico de Proceso, fijación de tolerancias estadísticas, análisis de series de tiempo, inspección,

Inspección, muestreo, histograma, diagrama e Ishikawa, diagrama de Pareto, graficas de dispersión, diseño de experimentos, prueba de hipótesis, metrología, análisis de la capacidad del proceso, análisis de regresión, análisis de confiabilidad, gráficos de Control Estadístico de Procesos; análisis de series de tiempo, hoja de verificación.

Diseño de experimentos, prueba de hipótesis, metrología, análisis de la capacidad del proceso, análisis de regresión, análisis de confiabilidad, muestreo, gráficos de Control estadístico de procesos, análisis de series de Tiempo, indicadores.

Muestreo, estadística descriptiva, hoja de verificación

Análisis de la capacidad del proceso, muestreo, gráficos de Contro estadístico de procesos, hoja de verificación, histograma, diagrama de Pareto. 


\section{Mejora}

10.1. Generalidades: La organización debe determinar y seleccionar las oportunidades de mejora e implementar cualquier acción necesaria para cumplir los requisitos del cliente y aumentar la satisfacción del cliente.

10.2. No conformidades y acciones correctivas: Cuando ocurra una no conformidad, incluidas las originadas por las quejas, la organización debe reaccionar a la no conformidad y cuando sea aplicable tomar acciones para controlarla, corregirla y hacer frente a las consecuencias.

10.3. Mejora continua: La organización debe mejorar continuamente la conveniencia, adecuación y eficacia del SGC.
Diseño de experimentos, prueba de hipótesis, análisis de la capacidad del proceso, análisis de regresión, muestreo; gráficos de control estadístico de procesos, análisis de series de tiempo.

Diseño de experimentos, prueba de hipótesis, análisis de la capacidad del proceso, análisis de regresión, muestreo; gráficos de control estadístico de procesos, análisis de series de tiempo. Diagrama Causa Efecto, Diagrama de Pareto, histograma, hoja de verificación.

Diseño de experimentos, prueba de hipótesis, análisis de la capacidad del proceso, análisis de regresión, muestreo; gráficos de control estadístico de procesos, análisis de series de tiempo. Diagrama Causa Efecto, Diagrama de Pareto, histograma.

\section{RESULTADOS}

Teniendo en cuenta el principio de Operación por procesos se hace indispensable controlar la producción y validar los procesos, con el fin de determinar los criterios y los métodos necesarios para asegurarse de que tanto la operación como el control de estos procesos sean eficaces. Además, la gestión de recursos se debe realizar con el fin de asegurar la disponibilidad de recursos e información necesarios para apoyar la operación y el seguimiento de estos procesos en pro de la gestión convenientemente de los productos no conformes, es decir, realizar el seguimiento y la medición cuando sea aplicable, para lo cual se pueden aplicar las siete herramientas de la calidad, estas comprenden: diagramas de Ishikawa que permiten determinar causas de los eventos de no calidad, hojas de registro para contabilizar la frecuencia de ocurrencia, los gráficos o cartas de control para verificar la estabilidad de los procesos, a la utilización de esta herramienta, [35] hace una contextualización para ajustar las cartas de control de acuerdo a las características de los procesos a partir de la perspectiva de autores como [36-39], quienes concuerdan en afirmar que los procesos no son tan ideales como lo muestra Shewhart ya que los métodos tradicionales de control estadístico pierden efectividad cuando se realizan pruebas con datos autocorrelacionados, en este trabajo se evidencia la aplicación de técnicas de muestreo, distribuciones de probabilidad y modelos de pronóstico para parametrizar procesos.

En cuanto a la planificación, diseño y desarrollo del producto se pueden utilizar, el Diseño concurrente (DC), esta es, según lo define [40], una metodología en la cual el diseño del producto y el proceso de fabricación están interrelacionados donde se realizan todas las actividades de diseño de manera paralela, integrando los propósitos de factores tales como cliente, proceso, integración interna/externa, gestión, producción seguridad y comunicación, consecuente con esta, para la determinación de los requisitos y expectativas del cliente, se requiere del QFD, pues el despliegue de la Función de Calidad resulta una herramienta muy precisa en transformar las expectativas del cliente en especificaciones de ingeniería, este es un método de diseño de productos y servicios que recoge las demandas y expectativas de los clientes y las traduce, en pasos sucesivos, a características técnicas y operativas satisfactorias, Según Michel Vigier, el QFD tiene como objetivo la obtención de una calidad de diseño excelente, mediante la conversión de las necesidades del cliente en características de calidad o "funciones de servicio" adecuadas, sin omisiones ni elementos superfluos[41], análisis del Valor (AV), esta metodología está enfocada en evaluar todas y cada una de las actividades que interviene en el procesos productivo a fin de establecer cuales aumentan valor y cuales solo aumentan costos [42], esto a partir control estadístico del Proceso (CEP) y análisis de modo de efecto de falla (AMEF) [11].

La Evaluación de desempeño, es realizar Seguimiento, medición del desempeño, análisis y evaluación tanto de los procesos como de la opinión del cliente, con el fin de Implementar las acciones necesarias para alcanzar los resultados planificados y la mejora continua de estos proceso, para esto, el diseño de experimentos puede contribuir a las síntesis de resultados, pues al analizar los cambios en el sistema bajo investigación y al evaluar estadísticamente el efecto se validan algunas características o se determina la influencia de uno o más 
factores sobre algunas características de un proceso, sistema o bien a la organización.

Por otro lado, el desempeño de una empresa también está condicionado o restringido por la capacidad del sistema, por lo cual, partiendo de la perspectiva de [43], la capacidad productiva, su análisis, planeación, programación y control, constituyen actividades críticas que se desarrollan paralelamente con las actividades de programación y planeación de materiales, siendo la capacidad la cantidad de productos o servicios destinados a satisfacer las necesidades del cliente o de la sociedad que puede ser obtenida por una unidad productiva en un determinado periodo de tiempo; para este análisis se hace necesario identificar las capacidad técnica, económica, instalada y la disponible [44], los modelos enfocados a este fin en su gran mayoría son problemas de programación lineal [45], en donde, la investigación de operaciones en el análisis, planeación, programación, control y evaluación de los procesos .

La mejora constituye el punto del ciclo PHVA donde se toman decisiones alrededor de la mitigación de las causas de las fallas en el sistema, por ende, las herramientas de ingeniería de la calidad para dar cumplimiento del ultimo numeral de la ISO 9001:2015, son las mismas descritas para la operación y la evaluación del desempeño, ya que al tener cuantificadas las causas de la no calidad, lo que sigue es la formulación de estrategias que las minimicen o si es posible que las eliminen.

\section{CONCLUSIONES}

El diseño, implementación, mantenimiento y mejora de un sistema de gestión de calidad bajo los lineamientos de la norma ISO 9001:2015, requiere de manera necesaria el uso y aplicación de la ingeniería de la calidad y por supuesto de las herramientas técnicas estadísticas con el fin de lograr una real eficiencia y no solo un proceso documental de cumplimiento de requisitos.

Siendo la ingeniería de la calidad un área en constante evolución, queda el espacio abierto para realizar futuros análisis e investigaciones sobre las herramientas estadísticas y su aplicación directa en los numerales de la norma ISO 9001:2015.

De acuerdo a la revisión realizada, se plantean unas herramientas necesarias en la planificación y operación de los procesos para la producción o prestación del servicio, tales como: el diseño de experimentos, la investigación de operaciones, la metrología y el análisis multivariado.

La evaluación de desempeño del sistema de gestión de calidad se hace más precisa con el uso de herramientas y técnicas de ingeniería de calidad, teniendo en cuenta que la recolección, el análisis y la evaluación de los datos se desarrolla bajo modelos y teorías aprobadas y reconocidas, permitiendo una eficiente toma de decisiones.

\section{REFERENCIAS}

[1] J. R.Evans, W.M. Lindsay, F.S. Fragoso, \& G.H. Díaz, Administración y Control de la Calidad: Thomson, 2000.

[2] W. A. Shewhart, Control económico de la calidad de productos manufacturados: Ediciones Díaz de Santos, 1997.

[3] V.E. Sower, Essentials of quality with cases and experiential exercises: John Wiley \& Sons, 2010.

[4] W.E. Deming, \& J.N. Medina, Calidad, productividad y competitividad: la salida de la crisis: Ediciones Díaz de Santos, 1989.

[5] J.M. Juran, Juran y la planificación para la calidad: Ediciones Díaz de Santos, 1990.

[6] A.V. Feigenbaum, M.A.G. De la Campa, M. A. G., \& P. Sevilla, Control total de la calidad (Vol. 1): Continental, 1994.

[7] A.J. Ruiz-Torres, J. Ayala-Cruz, N. Alomoto, \& J.L. Acero-Chavez, "Revisión de la literatura sobre gestión de la calidad: caso de las revistas publicadas en Hispanoamérica y España", "Estudios Gerenciales", vol. 31, $\mathrm{n}^{\circ}$. 136, pp. 319-334, 2015. DOI: https://doi.org/10.1016/j.estger.2015.01.004.

[8] D.H. Besterfield, \& V.G. Pozo, Control de calidad: Pearson Educación, 2009.

[9] ISO, I. O.f. S., ISO 9000 - Quality management, 2016. Disponible en: http://www.iso.org/iso/home/standards/management-standards/iso_9000.htm

[10] R.D.R. Bermúdez, “Ingeniería de calidad: robustez del proceso de urdido en una planta de likra por el método Taguchi," Revista Universidad EAFIT,vol. 29, n89,pp. 33-41, 2012.

[11] A. Zapata-Gómez, "Efecto de las técnicas de ingeniería de la calidad en el diseño de productos," Revista Ingeniería y Universidad, vol. 17, $\mathrm{n}^{\circ} 2$, $\mathrm{pp}$. 409-425, 2013.

[12] J.I.S. Committee, Japanese Industrial Standard. JIS K601, 1988. 
[13] K. Ishikawa, Introducción al control de calidad. España: Ediciones Díaz de Santos, 2007

[14] A.B. Godfrey, Juran's quality handbook, 1999.

[15] M. Cakmakci, N.M. Durakbasa, M.K. Karasu, G. Bas, \& U. Gurel, The importance of quality control within the relationship between the quality engineering and Taguchi methodology. Key Engineering Materials, 637, 2014. DOI:10.4028/www.scientific. net/KEM.637.27.

[16] J.M. Juran, F,M. Gryna, \& R.S. Bingham, Manual de Control de la Calidad: Reverté, 1983.

[17] G. Taguchi, S. Chowdhury, \& Y. Wu, Taguchi's quality engineering handbook: Wiley-Interscience, 2005.

[18] I. F. D. Aguirre, "Introducción a la Ingeniería Robusta". Dyna, vol. 81, $n^{\circ}$ 5, pp. 29-32, 2006.

[19] B. L. Hansen, \& P.M. Ghare, Control de calidad: teoría y aplicaciones. España: Ediciones Díaz de Santos, 2008

[20] J.D. Terán Ruales, Implementación del sistema de gestión de aseguramiento y control de calidad en el departamento técnico para los productos y servicios DE COHECO SA, 2014.

[21] Hernández Pedrera, C., \& Da Silva Portofilipe, F. (2016). Aplicación del control estadístico de procesos (CEP) en el control de su calidad. Tecnología Química, 36(1), 104-116.

[22] P. Crosby, P., Reflexiones sobre calidad : 295 máximas del gurú mundial de calidad Philip Crosby: McGraw-Hill Interamericana, 2000.

[23] ISO, I. O. f. S. ISO/TR 10017. Orientación Sobre Técnicas Estadísticas, 2003.

[24] K. Ishikawa, Qué es el control total de calidad?: la modalidad japonesa: Editorial Norma, 1997.

[25] J.B.D.I. Casas, "El ISO 9001 Y TQM en las empresas Latinoamericanas: Perú". Globalización, Competitividad y Gobernabilidad de Georgetown/Universia, 8,1, 2014. DOI: 10.3232/GCG.2013.V8.N1.04.

[26] C. Chow-Chua, M. Goh, \& T. Boon Wan, Does ISO 9000 certification improve business performance? International Journal of Quality \& Reliability Management, vol. 20, nº 8, pp. 936-953, 2003. DOI: https:// doi.org/10.1108/02656710310493643.

[27] P. Gejdoš, "Continuous Quality Improvement by Statistical Process Control,". Procedia Economics and Finance, 34, 565-572, 2015. DOI: https://doi. org/10.1016/S2212-5671(15)01669-X.

[28] ISO., I. O. f. S. Management system standards, 2016. from http://www.iso.org/iso/home/standards/management-standards.htm
[29] P. Padma, L. Ganesh, \& C. Rajendran, "A study on the critical factors of ISO 9001: 2000 and organizational performance of Indian manufacturing fir$\mathrm{ms}$,". International Journal of Production Research, vol. $46 n^{\circ} 18$, pp. 4981-5011, 2008. DOI: https:// doi.org/10.1080/00207540600793299.

[30] ISO, I. O. f. S. ISO 9000:2015 Quality management systems - Fundamentals and vocabulary, 2015. from: $\quad$ https://www.iso.org/obp/ui/\#iso:std:iso:9000:ed-4:v1:en

[31] ISO_9001, ISO 9001:2015 Sistemas de Gestión de la Calidad. Requisitos. Colombia: ICONTEC, 2015

[32] ISO_31000. NTC ISO 31000. GESTIÓN DEL RIESGO. PRINCIPIOS Y DIRECTRICES. Colombia: ICONTEC, 2011.

[33] S.T. Meegan, \& W.A. Taylor, "Factors influencing a successful transition from ISO 9000 to TQM: the influence of understanding and motivation,". International Journal of Quality \& Reliability Management, 14(2), 100-117, 1997. DOI: https://doi. org/10.1108/02656719710165383.

[34] M.Terziovski, D. Samson, \& D. Dow, The business value of quality management systems certification. Evidence from Australia and New Zealand. Journal of oper ations management, 15(1), 1-18, 1997. DOI: https://doi.org/10.1016/S0272-6963(96)00103-9.

[35] R.P. Niebles, Ó Oviedo-Trespalacios, J.G.V. Cabeza, \& L.M.F. Cantillo, L. M. F., "Diseño estadístico de cartas de control para datos autocorrelacionados,". Ingeniería y Desarrollo, vol. 31, $n^{\circ} 2$, pp. 291-315, 2013.

[36] D.C. Montgomery, \& C.M. Mastrangelo, Some statistical process control methods for autocorrelated data. Journal of Quality Technology, vol. 23, $\mathrm{n}^{\circ} 3$, pp. 179-193, 1991.

[37] T. Sukchotrat, S.B. Kim, \& F.Tsung, "One-class classification-based control charts for multivariate process monitoring,". IIE transactions, vol. $42 n^{\circ}$ 2, pp. 107-120, 2009. DOl:http://dx.doi. org/10.1080/07408170903019150.

[38] S. Vander Wiel, S., "Monitoring processes that wander using integrated moving average models,". Technometrics, vol 38, n² 2, pp. 139-151, 1996. DOI: https://doi.org/10.1080/00401706.1996.104 84459.

[39] N.F. Zhang, "A statistical control chart for stationary process data,". Technometrics, vol. $40, \mathrm{n}^{\circ} 1$, pp. 24-38, 1998. DOI: https://doi.org/10.1080/004 01706.1998.10485479.

[40] R. Kinna, "Teamworking and concurrent engineering-a success story,". World class design to manu- 
facture, vol. 2, $n^{\circ}$ 3, pp. 5-9, 1995. DOI: https://doi. org/10.1108/09642369310087048.

[41] E. Yacuzzi, \& F. Martín, QFD: Conceptos, aplicaciones y nuevos desarrollos: Serie Documentos de Trabajo, Universidad del CEMA: Área: negocios, 2003.

[42] Fowler, T. C. (1990). Value analysis in design: CRC Press.

[43] M. Dominguez, \& A. Jose, Dirección de Operaciones: Aspectos tácticos y operativos en la producción y los servicios: MCGRAW HILL, 1995.
[44] D. Kalenatic,Modelo integral y dinámico para el análisis, planeación, programación y control de las capacidades productivas en empresas manufactureras: Universidad Distrital Francisco José de Caldas, Centro de Investigaciones y Desarrollo Científico, Instituto de Investigaciones Científicas, 2001.

[45] D. Kalenatic, C.A.L. Bello, \& L.J.G. Rodríguez, Modelo de Medición, Análisis, Planeación y Programación de Capacidades en un Contexto de Múltiples Criterios de Desición. Ingeniería, 10(2), 57-66, 2005. 